\title{
Evaluación de la longitud de los telómeros en caballos, mediante el uso de la reacción en cadena de la polimerasa cuantitativa
}

\author{
Sanmartín-Sánchez L. ${ }^{\text {a }}$, Perea-Muñoz JM. ${ }^{2 b}$, Pérez-Rico A. ${ }^{\text {a }}$, Vega-Pla JL. ${ }^{3 a}$
}

Sanid. mil. 2014; 70 (2): 71-75; ISSN: 1887-8571

\begin{abstract}
RESUMEN
Introducción: Los telómeros son estructuras de los cromosomas eucariotas, que juegan un importante papel en el mantenimiento de la estabilidad e integridad celular, estableciéndose como uno de los posibles biomarcadores del envejecimiento celular en el hombre y otras especies. Objetivos: Evaluar la posibilidad del uso de la técnica de la reacción en cadena de la polimerasa cuantitativa (qPCR) para calcular la longitud relativa de los telómeros en caballos y asociarla con factores como la edad, la raza y el y el sexo. Material y métodos: Se amplificó una secuencia repetitiva correspondiente a los telómeros, junto con otra de un gen de copia única (Interleukina-2), en muestras de sangre de 175 caballos de tres razas, de distintos grupos de edad y de ambos sexos. Se calculó la longitud relativa de los telómeros de cada individuo a través dos variables de respuesta: la diferencia entre el número de ciclos de cuantificación entre telómeros e Interleukina-2 (Dif Cq) y la ratio entre ambos valores ( $T / S$ ratio). Resultados: La variable Dif $C q$ presenta una asociación más fuerte con la edad que la variable $T / S$ ratio. Se observó una correlación negativa entre la edad y la longitud de los telómeros $(\mathrm{R}=-0,31, \mathrm{p}<0,05)$. Conclusión: La técnica de qPCR es aplicable para determinar la longitud relativa de los telómeros en caballos. Los individuos mayores de 20 años presentan telómeros acortados, aunque no se encontró un efecto significativo ni de la raza, ni del sexo.
\end{abstract}

PALABRAS CLAVE: Caballo, telómero, Reacción en Cadena de la Polimerasa cuantitativa (qPCR), edad, raza, sexo.

Assessment of the length of horse telomeres using the quantitative polymerase chain reaction

SUMMARY: Introduction: Telomeres are specialized structures at the ends of eukaryotic chromosomes which play an important role in the maintenance of cell integrity and stability. The telomere length is considered one of the potential biomarkers of cellular aging. Objectives: To evaluate the possible use of quantitative polymerase chain reaction qPCR in the estimation of relative length of equine chromosome telomeres. Material and methods: A repetitive sequence corresponding to telomeres and a single copy gene (Interleukin-2) were amplified from blood samples of 175 horses of three breeds, different ages and both sexes. The relative length of telomeres of each individual was calculated by means of the response variables: the difference of quantification cycles number between telomeres and Interleukin-2 (Dif Cq) and the ratio between both values ( $T / S$ ratio). Results: The variable Dif $C q$ has a stronger association with the age than the variable $T / S$ ratio. There was a negative correlation between the age and telomere length $(\mathrm{R}=-0,31$, $\mathrm{p}<0,05)$. Conclusion: The qPCR technique is applicable to determine the relative length of telomeres in horses. The length of the telomeres shorten significantly from age 20, although no effect was found over the breed or sex.

KEY WORDS: Horse, telomere, Quantitative Polimerase Chain Reaction (qPCR), age, breed, sex.

\section{INTRODUCCIÓN}

Los telómeros son estructuras cromatínicas especializadas que se encuentran localizadas en los extremos de los cromosomas de la células eucariotas. El ADN telomérico (ADNt) está constituido por repeticiones en tándem de pequeñas secuencias nucleotídicas (TTAGGG) con una distribución asimétrica de los pares de bases de Guanina y Citosina ${ }^{1}$. Están implicados en muchas funciones celulares, especialmente las relacionadas con el

${ }^{1}$ Tte. Veterinario.

2 Profesor contratado doctor.

${ }^{3}$ Tcol. Veterinario.

a Laboratorio de Investigación Aplicada. Cría Caballar de las Fuerzas Armadas. Córdoba. España.

${ }^{\mathrm{b}}$ Universidad de Córdoba. Dpto. de Producción Animal. Córdoba. España.

Dirección para correspondencia: Jose Luis Vega Pla. Laboratorio de Investigación Aplicada, Apartado de Correos 2087, 14080-Córdoba. Telf.: 957325312 Fax.: 957322493. Correo: jvegpla@oc.mde.es.

Recibido: 20 de mayo de 2013

Aceptado: 18 de octubre de 2013 control de la longevidad de las diferentes estirpes celulares y en otras como la mitosis. Los telómeros protegen el final de los cromosomas aportando estabilidad durante la replicación celular ${ }^{2}$, juegan también un papel fundamental en la respuesta celular al estrés y en consecuencia, sobre el daño directo al DNA ${ }^{3}$.

Se han puesto de manifiesto indicios del acortamiento de los telómeros asociadas directamente con el envejecimiento ${ }^{4}$. De hecho, se ha sugerido que este fenómeno sea la principal causa de senescencia celular prematura ${ }^{5,6}$, como se muestra en numerosos estudios, tanto en la especie humana ${ }^{7-9}$, como en algunas especies animales ${ }^{10-12}$. Así, Cawthon y col. ${ }^{13}$ encontraron correlación positiva entre el riesgo de muerte y el acortamiento de los telómeros de personas mayores de 60 años. Otros estudios también han puesto de manifiesto correlaciones positivas entre el acortamiento de los telómeros y una gran variedad de enfermedades relacionadas con la edad ${ }^{14-18}$. Este hecho podría ser muy significativo para el diseño de planes de selección de reproductores en el mundo equino. Además, dado que los mecanismos subyacentes, así como los factores que influyen en la variación de la longitud de los telómeros, dentro y entre razas son poco conocidos, resulta 


\section{Sanmartín Sánchez, et al.}

de interés avanzar en el estudio de la influencia de otros factores como, la raza y el sexo.

Entre los métodos que se utilizan para la medición de la longitud de los telómeros destacan: el análisis de fragmentos de restricción terminales (TRF); la prueba de citometría de flujo de células y fluorescencia in situ (Flow-FISH); el análisis de fluorescencia mediante hibridación in situ (FISH) con un polímero artificial similar al ADN (PNA) y la reacción en cadena de la polimerasa cuantitativa (qPCR). Con la excepción del ensayo de TRF, todos los métodos generan una medida relativa de la longitud de los telómeros ${ }^{19-21}$. Un procedimiento para calcular la longitud de los telómeros mediante qPCR es el propuesto por Cawthon y col. ${ }^{19}$ que consiste en obtener una medida relativa de la longitud mediante la amplificación de una secuencia repetitiva (TGAGGG) propia de los telómeros en mamíferos, usando un gen de copia única como calibrador de la amplificación. Las ventajas de usar la qPCR es que, a diferencia de los ensayos basados en la hibridación de sondas, se requieren pequeñas cantidades de $\mathrm{ADN}$ y se pueden procesar un gran número de muestras simultáneamente. Sin embargo, en muestras equinas todavía no ha sido utilizada como herramienta para estimar la longitud de los telómeros.

Se plantea como objetivo estudiar la longitud de los telómeros en células sanguíneas mediante la técnica qPCR, en caballos Pura Sangre Inglés, Pura Raza Árabe y Pura Raza Español de ambos sexos y diferentes edades.

\section{MATERIAL Y MÉTODOS}

Se seleccionaron 175 caballos de las razas Pura Raza Española (PRE) Pura Raza Árabe (PRá) y Pura Sangre Inglés (PSI), procedentes del Organismo Autónomo Cría Caballar de las Fuerzas Armadas del Ministerio de Defensa (tabla 1).

Tabla 1. Distribución de la muestra en función de la raza, sexo, edad.

\begin{tabular}{|lccccc|}
\hline Raza & Sexo & $<1$ año & 9 años & $\mathbf{2 1 - 2 8}$ años & Total \\
\hline PRE & Hembra & 9 & 10 & 10 & 29 \\
& Macho & 10 & 10 & 9 & 29 \\
PRá & Hembra & 9 & 10 & 9 & 28 \\
& Macho & 10 & 10 & 9 & 29 \\
PSI & Hembra & 10 & 10 & 19 & 39 \\
& Macho & 10 & 10 & $1^{*}$ & 21 \\
\hline Total & & 58 & 60 & 57 & 175 \\
\hline
\end{tabular}

PRE: Pura Raza Español; PRá: Pura Raza Árabe; PSI: Pura Sangre Inglés. 1 * Sólo fue posible disponer de una muestra para su análisis.

Las muestras se procesaron según un protocolo estándar para la extracción del ADN. Se amplifican las secuencias teloméricas y como calibrador se usó el gen de la Interleukina-2 (IL-2).

La PCR cuantitativa (en inglés, quantitative polymerase chain reaction; qPCR) o PCR en tiempo real, es una variante de la reacción en cadena de la polimerasa (PCR) utilizada para amplificar y simultáneamente cuantificar de forma absoluta el producto de la amplificación de ácido desoxirribonucleico (ADN). Para ello emplea, del mismo modo que la PCR convencional, un molde de ADN, al menos un par de cebadores específicos,
dNTPs, un tampón de reacción adecuado, y una ADN polimerasa termoestable; a dicha mezcla se le adiciona una sustancia marcada con un fluoróforo que, en un termociclador que albergue sensores para medir fluorescencia tras excitar el fluoróforo a la longitud de onda apropiada, permita medir la tasa de generación de uno o más productos específicos

La composición de las reacciones de qPCR para la secuencia de telómeros e $I L-2$ fueron idénticas, excepto para la composición oligonucleotídica de los cebadores. Las concentraciones de reactivos utilizadas corresponden a :1\% EvaGreen (BіотUм); Tris-HCl, pH 8, 15 mM; KCl 50 mM; MgCl2 3 mM; dNTP 0,2 mM; MyTaq (Bioline) 0,5 U. La concentración final de los cebadores fue de 0.3 $\mu \mathrm{M}$. Las secuencias de los cebadores de telómeros $\left(5^{\prime} \rightarrow 3^{\prime}\right)$ fueron: Tell: GGT TTT TGA GGG TGA GGG TGA GGG TGA GGG TGA GG GT; Tel2: TCC CGA CTA TCC CTA TCC CTA TCC CTA TCC CTA TCC CTA; y para la $I L-2$ fueron $I L-2$ A: GGG AAA CAC AGC AAC TG; $I L-2$ B: GCC TTC TTG GGG ATG TTA AT. Todas las amplificaciones se realizaron en un termociclador Rotor-Gene 6000 (Corbett Research Ltd, Cambridge, UK). Se programó un primer ciclo de activación de la polimerasa de 95 ${ }^{\circ} \mathrm{C}$ durante $1 \mathrm{~min}, 40$ ciclos de $95^{\circ} \mathrm{C} 20 \mathrm{~s}, 60{ }^{\circ} \mathrm{C} 30$ s y $72{ }^{\circ} \mathrm{C} 20 \mathrm{~s}$, haciendo lecturas de fluorescencia en el último paso de cada ciclo térmico. El uso de muchos ciclos en qPCR es habitual para comprobar que la reacción de control sin muestra no amplifica inespecíficamente o lo hace muy tarde. Finalmente, se sometieron las muestras a un incremento progresivo de temperatura para calcular el punto de fusión de los fragmentos amplificados (desde $60^{\circ} \mathrm{C}$ hasta $95^{\circ} \mathrm{C}$, elevándose la temperatura $1{ }^{\circ} \mathrm{C}$ en cada paso de $5 \mathrm{~s}$ ).

Los datos brutos de cada $\mathrm{qPCR}$ se procesaron mediante el módulo de análisis Comparative Quantitation (Rotor-Gene 6000 software, Corbett Research Ltd, Cambridge, UK). Finalmente, se realizó un análisis de las curvas de fusión para comprobar que las amplificaciones generaron fragmentos con características similares en cuanto a su composición. Asimismo, se tipificó una misma muestra en todas las tandas como calibrador y compensar las diferencias entre experimentos.

Adicionalmente, algunas muestras fueron sometidas a una electroforesis en un gel de agarosa al 1\% para observar si el tamaño de los fragmentos generados en la amplificación era el esperado.

Se determinó la diferencia entre el Ciclo de Cuantificación (Cq) de las secuencias teloméricas con respecto al Cq del gen de copia única para cada muestra de $\operatorname{ADN}($ Dif $C q$ ). El Cq es el número de ciclos necesarios para que se produzca un aumento de fluorescencia significativo con respecto a la señal de base, y es inversamente proporcional a la cantidad inicial de moléculas molde. Dado que la cantidad de producto amplificado se duplica aproximadamente en cada ciclo, la longitud relativa de los telómeros se puede determinar según la fórmula del $T / S$ ratio propuesta por Cawton y col. ${ }^{19}$

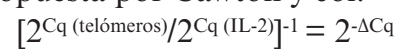

Donde $C q$ (telómeros) es el ciclo de cuantificación de las secuencias teloméricas de un animal concreto, $C q(I L-2)$ es el ciclo de cuantificación de la Interleukina 2 para la misma muestra.

La reproducibilidad de la cuantificación se probó mediante la amplificación de 24 muestras elegidas aleatoriamente. Se realizó un análisis de regresión lineal entre los $\mathrm{Cq}$ de las muestras del día de los análisis iniciales y los Cq correspondientes a las mismas muestras analizadas otro día distinto. 


\section{Análisis estadístico}

Los caballos fueron agrupados atendiendo a su edad en potros de temprana edad ( $<1$ año), adultos de 9 años y adultos de avanzada edad (21-28 años). Se agruparon también atendiendo a la raza (Pura Raza Español, Pura raza Árabe, Pura Sangre Inglés) y al sexo (machos y hembras). Se calcularon dos variables de respuesta indicativas de la longitud relativa de los telómeros de cada muestra: la Diferencia entre los Ciclos de Cuantificación de ambos genes (Dif Cq); y la T/S ratio propuesta por Cawton y col. ${ }^{19}$

Se contrastó la normalidad y la homocedasticidad de los valores obtenidos para ambas variables, mediante el test de Shapiro y el contraste $\mathrm{C}$ de Cochran respectivamente. Se utilizó un modelo de Análisis de la Varianza de tipo factorial (ANOVA factorial) para valorar la influencia del efecto de la edad, la raza y el sexo sobre la de la longitud de los telómeros medida como la Dif $C q$. Se empleó el test de Student Newman Keuls (SNK), para comprobar las interacciones entre grupos. Para la variable T/S ratio, se utilizó la técnica no paramétrica de Kruskall-Wallis, y se compararon los grupos de edad dos a dos mediante el test Mann-Whitney. La correlación entre ambas variables de respuesta y la edad fue modelada mediante el test de correlación de Pearson. Se consideró un $p$-value menor o igual a 0,05. Para los análisis estadísticos se utilizó el software Statgraphics Plus Professional 16.0.03.

\section{RESULTADOS}

La amplificación de las secuencias de los telómeros se puso de manifiesto entre los ciclos 6 y 20 de la qPCR. En el caso de la amplificación del gen de copia única $(I L-2)$, las muestras amplificaron entre los ciclos 19 y 23 (figura 1).

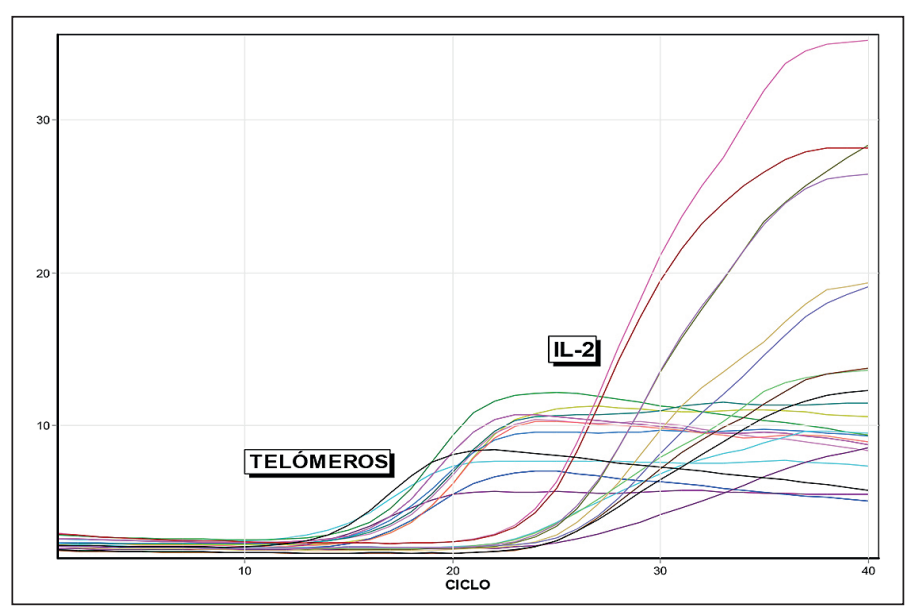

Figura 1. Productos obtenidos mediante $q P C R$ de algunos individuos. Cada pareja de colores corresponde a ambos productos génicos obtenidos de la amplificación de la misma muestra. Las amplificaciones correspondientes a los telómeros tienen unos valores de ciclo umbral inferiores, mientras que las correspondientes al gen de copia única son mayores, constantes y de baja variabilidad.

En la electroforesis (figura 2) el gen de copia única (IL-2) se manifestó como una banda única de peso molecular constante

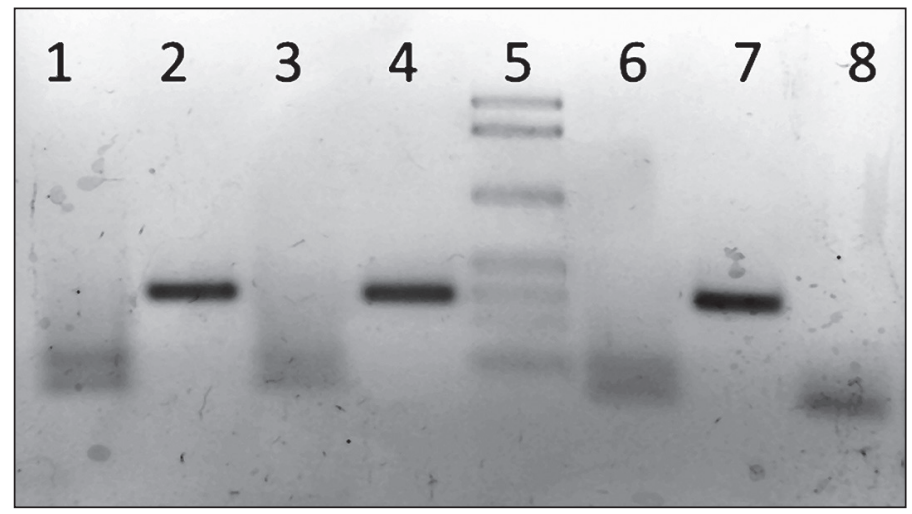

Figura 2. Electroforesis en gel de agarosa de los productos de amplificación. El sentido de la migración es de arriba hacia abajo. Secuencias teloméricas (columnas 1; 3 y 6); gen de copia única (columnas 2; 4 y 7) y agua (columna 8). Marcador de pesos moleculares $(706 ; 608 ; 406 ; 269 ; 215 ; 170 ; 118)$ pares de bases (columna 5).

(215 pares de bases), mientras que los telómeros se observaron como una mancha, dado que presentaron fragmentos de diferentes longitudes.

El análisis de la reproducibilidad reveló linealidad significativa entre las medidas de $\mathrm{Cq}$ obtenidas en diferentes ensayos $(\mathrm{R}$ $=0,90$ y $0,83, \mathrm{p}=0,00$ y $\mathrm{p}=0,00$, para telómeros e $I L-2$, respectivamente). El coeficiente de variación medio en las medidas repetidas fue de $1,52 \%$ y $2,07 \%$, respectivamente.

Los valores obtenidos de las variables de respuesta, Dif $C q$ y el $T / S$ ratio se organizaron según los grupos definidos por los factores de estudio (edad, raza y sexo). La variable Dif $C q$ mostró buen ajuste a la distribución normal $(\mathrm{W}=0,98, \mathrm{p}=0,86)$ (Test de Shapiro ), sin embargo la variable $T / S$ ratio no se ajustó a una distribución normal ( $\mathrm{W}=0,42, \mathrm{p}=0,00$ ).

En la tabla 2 se muestran las medias y desviaciones típicas de la variable $D$ if $C q$ en cada grupo. Para cuantificar el efecto de la edad, la raza y el sexo sobre la variable Dif $C q$ se utilizó un modelo de Análisis de Varianza Factorial. Previamente, se contrastó la igualdad de varianzas mediante el contraste $\mathrm{C}$ de Cochran $(\mathrm{C}=0,42, \mathrm{p}=0,16)$. El análisis de varianza mostró (tabla 3) que sólo la edad afectó significativamente a la longitud de los telómeros $(F=5,24 ; p<0,05)$. Se utilizó el test de Student-Newman-Keuls (SNK) para determinar la existencia de diferencias significativas entre los grupos de edad (figura 3). El test SNK indicó que los grupos 1 ( $<1$ año) y 2 (9 años) fueron homogéneos y significativamente superiores $(\mathrm{p}<0,05)$ al grupo 3 (21 a 28 años).

Tabla 2. Descripción estadística de la variable Dif Cq (media \pm desviación típica).

\begin{tabular}{|cccccc|}
\hline Raza & Sexo & $<1$ año & 9 años & 21-28 años & Media \\
\hline PRE & Hembra & $9,83( \pm 1,27)$ & $9,96( \pm 0,83)$ & $8,59( \pm 1,87)$ & $9,46( \pm 0,76)$ \\
& Macho & $9,54( \pm 1,26)$ & $10,50( \pm 0,65)$ & $8,57( \pm 2,22)$ & $9,54( \pm 0,97)$ \\
PRá & Hembra & $10,14( \pm 1,21)$ & $9,84( \pm 1,04)$ & $9,41( \pm 1,30)$ & $9,79( \pm 0,37)$ \\
& Macho & $9,58( \pm 1,61)$ & $9,34( \pm 1,55)$ & $9,41( \pm 1,30)$ & $9,44( \pm 0,12)$ \\
PSI & Hembra & $9,88( \pm 1,56)$ & $9,76( \pm 1,01)$ & $9,41( \pm 1,30)$ & $9,68( \pm 0,24)$ \\
& Macho & $10,18( \pm 1,96)$ & $9,28( \pm 1,03)$ & $8,80( \pm 0,00)$ & $9,42( \pm 0,70)$ \\
\hline Total & - & $9,86( \pm 0,27)$ & $9,78( \pm 0,45)$ & $9,03( \pm 0,42)$ & \\
\hline
\end{tabular}

PRE: Pura Raza Español; PRá: Pura Raza Árabe; PSI: Pura Sangre Inglés. 
Tabla 3. Análisis de Varianza Factorial: efecto de la raza, sexo y edad sobre la variable Dif $C q$.

\begin{tabular}{|lccccc|}
\hline & $\begin{array}{c}\text { Suma de } \\
\text { cuadrados }\end{array}$ & $\begin{array}{c}\text { Grados } \\
\text { de } \\
\text { libertad }\end{array}$ & $\begin{array}{c}\text { Cuadrado } \\
\text { Medio }\end{array}$ & Cociente-F & P-value \\
\hline \multicolumn{2}{|l}{ Efectos principales } & & & & \\
A: edad & 20,60 & 2 & 10,30 & 5,24 & 0,01 \\
B: raza & 0,12 & 2 & 0,06 & 0,03 & 0,96 \\
C: sexo & 0,86 & 1 & 0,86 & 0,44 & 0,50 \\
Interacciones & & & & & \\
AB & 9,60 & 4 & 10,30 & 5,24 & 0,30 \\
AC & 0,12 & 2 & 0,06 & 0,03 & 0,99 \\
BC & 3,16 & 2 & 1,58 & 0,81 & 0,44 \\
ABC & 3,29 & 4 & 0,82 & 0,42 & 0,79 \\
\hline
\end{tabular}

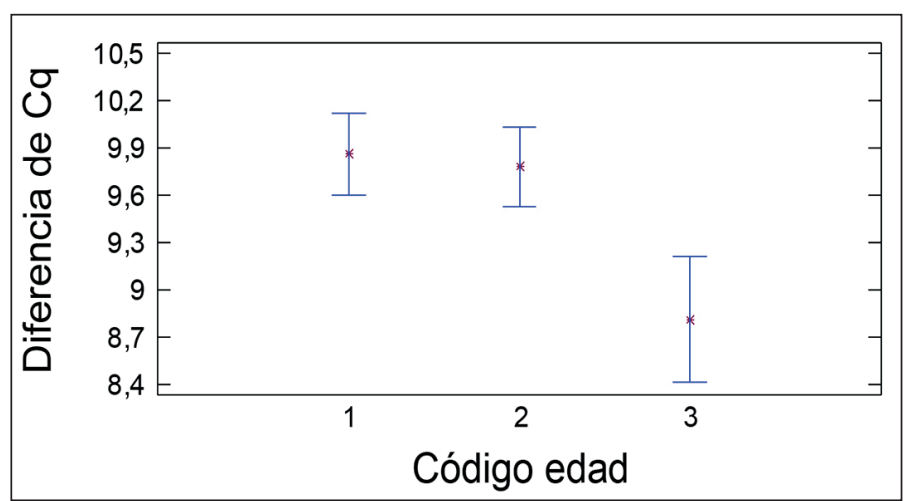

Figura 3. Intervalos de confianza para las medias de la variable Dif Cq en los tres grupos de edad evaluados (Abreviaturas de Código edad; 1: < 1 año; 2: 9 años; 3: 21-28 años).

El test de Kruskal-Wallis reveló que sólo la edad afecta de modo significativo al $T / S$ ratio $(\mathrm{p}<0,05)$. La longitud de los telómeros medida mediante el $T / S$ ratio es similar y significativamente inferior $(\mathrm{p}<0,05)$ en los grupos 2 (9 años) y 3 ( 21 a 28 años) aplicando el test no paramétrico de Mann-Whitney (figura 4).

La correlación de Pearson entre Dif $C q$ y $T / S$ ratio, fue de 0,64 $(\mathrm{p}=0,00)$, mientras que la correlación entre la edad y la $D$ if $C q$ y el $T / S$ ratio fue de $-0,31(\mathrm{p}=0,00)$ y $-0,16,(\mathrm{p}=0,00)$, respectivamente

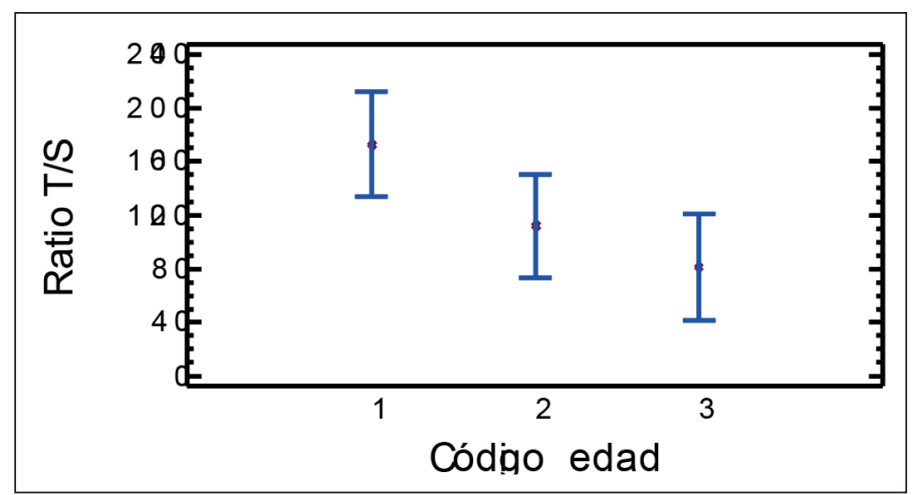

Figura 4. Intervalos de confianza para las medias de la variable $T / S$ ratio en los tres grupos de edad evaluados mediante el test no paramétrico de Mann-Whitney (Abreviaturas de Código edad; 1: <1 año; 2: 9 años; 3: 21-28 años).

\section{DISCUSIÓN}

En el presente trabajo se han mostrado indicios de que los telómeros de las células sanguíneas de la serie blanca de caballos (Equus caballus), se acortan con la edad, efecto que se ha podido revelar mediante la técnica qPCR. Asimismo, también se ha estudiado la variación de la longitud relativa de los telómeros en tres razas equinas y en ambos sexos, aunque sin obtener resultados significativos.

La qPCR simplifica enormemente el cálculo relativo de la longitud de los telómeros ${ }^{19-21}$. Sin embargo, en los resultados obtenidos se ha observado que pequeñas variaciones en el ciclo de cuantificación (Cq) se magnifican cuando se usa el $T / S$ ratio para el cálculo de la longitud. La comparación de ambas variables ha mostrado que aunque la variable $T / S$ ratio sea un indicador directo de la longitud relativa, no es un buen estimador del acortamiento relacionado con la edad respecto a la variable Dif $C q$. Estos resultados están en línea con los de O'Callaghan y Fenech ${ }^{21}$, quienes obvian la variable $T / S$ ratio propuesta por Cawton y col. ${ }^{19}$ obteniendo mejores estimaciones mediante la técnica de qPCR y el cálculo absoluto de la longitud de los telómeros

Se ha demostrado que la longitud de los telómeros se estabiliza en los individuos de mediana edad y se acorta en los de mayor edad. Estos hallazgos están en la misma línea que los mostrados por Aubert y col. ${ }^{3}$ y Kateppali y col. ${ }^{22}$ en 2008.

Se ha sugerido que el mantenimiento de la estructura de los telómeros se asocia con la mortalidad tardía ${ }^{23}$. Esto podría constituir un biomarcador en individuos jóvenes y de mediana edad. En este intento de conocer la longitud de los telómeros en las tres principales razas de Cría Caballar, no se han encontrado diferencias significativas. Podría ser que la baja variabilidad genética descrita entre las diferentes razas de caballos $^{24-26}$, sumada a la amplia variabilidad detectada en la longitud relativa de los telómeros en los leucocitos, dificulte el uso de este tipo de células para encontrar diferencias entre razas en base a la longitud de los telómeros. Por otra parte, la longitud de los telómeros se ha estudiado en una gran población humana no encontrándose diferencias significativas entre hombres y mujeres ${ }^{27}$ resultado que coincide con el obtenido en el presente trabajo donde tampoco se han podido establecer estas diferencias entre caballos y yeguas.

\section{CONCLUSIONES}

La edad se correlaciona negativamente con la longitud de los telómeros de las células sanguíneas de la serie blanca de la especie equina, mientras que no se han encontrado efectos significativos ni del sexo ni de la raza empleando la técnica de qPCR.

La variable Dif $C q$, como indicador de la longitud relativa de los telómeros, se correlaciona mejor con la edad que la variable T/S ratio.

\section{AGRADECIMIENTOS}

Al equipo técnico del Laboratorio de Investigación Aplicada de Cría Caballar por su excelente profesionalidad, en es- 
pecial al Brigada EQSUB/VAV Francisco Ruiz Pacheco por su labor técnica.

El estudio se encuadra dentro de las actividades y objetivos del Convenio de Colaboración que el Organismo Autónomo Cría Caballar de las Fuerzas Armadas tiene suscrito con la Diputación de Córdoba. El Organismo Autónomo Cría Caballar de las Fuerzas Armadas ha financiado los análisis de biología molecular. La Diputación de Córdoba ha contribuido prestando sus instalaciones para realizar los análisis genéticos.

\section{BIBLIOGRAFIA}

1. Lansdorp PM, Verwoerd FM, van de Rijke, Dragowska V, Little MT, Dirks RW, Raap AK, Tanke HJ. Heterogeneity in telomere length of human chromosomes. Hum Mol Genet 1996; 5:685-691.

2. Muller HJ. The re-making of chromosomes. Collecting Net, Woods Hole 1938; 13: 181-98

3. Aubert G, Lansdorp PM. Telomeres and Aging. Physiol Rev 2008; 88: 557-79.

4. Cooke HJ, Smith BA. Variability at the telomeres of the human X/Y pseudoautosomal region. Cold Spring Harb Symb Quant Biol 1986; 51: 213-9.

5. Allsopp RC, Vaziri H, Patterson C, Goldstein S, Younglai EV, Futcher AB, Greider CW, Harley CB. Telomere length predicts replicative capacity of human fibroblasts. Proc Natl Acad Sci USA 1992; 89: 10114-8.

6. Harley CB, Vaziri H, Counter CM, Allsopp RC. The telomere hypothesis of cellular aging. Exp Gerontol 1992; 27: 375-82.

7. Jemielity S, Kimura M, Parker KM, Parker JD, Cao X, Aviv A, et al. Short telomeres in short-lived males: what are the molecular and evolutionary causes?. Aging Cell 2007;6(2):225-33.

8. Blasco MA. Telomeres and human disease: ageing, cancer and beyond. Nat Rev Genet 2005; 6(8):611-22.

9. Parwaresch R, Krupp G. Telomere biology and the molecular basis of aging. Arkh Patol 2002; 64(3):37-9.

10. Jeyapalan JC, Ferreira M, Sedivy JM, Herbig U. Accumulation of senescent cells in mitotic tissue of aging primates. Mech Ageing Dev 2007; 128(1):36-44.

11. Swanberg SE, Delany ME. Differential expression of genes associated with telomere length homeostasis and oncogenesis in an avian model. Mech Ageing Dev 2005; 126(10):1060-70.
12. Argyle D, Ellsmore V, Gault EA, Munro AF, Nasir L. Equine telomeres and telomerase in cellular immortalisation and ageing. Mech Ageing Dev 2003; 124(6):759-64.

13. Cawthon RM, Smith KR, O’Brien E, Sivatchenko A, Kerber RA. Association between telomere length in blood and mortality in people aged 60 years or older. Lancet 2003; 361(9355):393-5.

14. Goronzy JJ, Fujii H, Weyand CM. Telomeres, immune aging and autoimmunity. Exp Gerontol 2006; 41(3):246-51.

15. Brouilette S, Singh RK, Thompson JR, Goodall AH, Samani NJ. White cell telomere length and risk of premature myocardial infarction. Arterioscler Thromb Vasc Biol 2003; 23(5):842-6.

16. Von Zglinicki T. Role of oxidative stress in telomere length regulation and replicative senescence. Ann NY Acad Sci 2000; 908:99-110.

17. Benetos A, Okuda K, Lajemi M, Kimura M, Thomas F, Skurnick J, et al.Telomere length as an indicator of biological aging: the gender effect and relation with pulse pressure and pulse wave velocity. Hypertension 2001; 37(2):381-5.

18. Samani NJ, Boultby R, Butler R, Thompson JR, Goodall AH. Telomere shortening in atherosclerosis. Lancet 2001; 358(9280):472-3.

19. Cawthon RM. Telomere measurement by quantitative PCR. Nucleic Acids Research 2002: 30(10):e47.

20. O'Callaghan N, Dhillon V, Thomas P, Fenech M. A quantitative real-time PCR method for absolute telomere length. BioTechniques 2008; 44(6):807-809.

21. O'Callaghan N, Fenech M.A quantitative PCR method for measuring absolute telomere length. Biological Procedures Online 2011; 13:3.

22. Katepalli MP, Adams AA, Lear TL, Horohov TL. The effect of age and telomere length on immune function in the horse. Dev Comp Immunol 2008; 32:1409-15.

23. Tilesi F, Di Domenico EG, Pariset L, Bosco L, Willems D, Valentini A, Ascenzioni F. Telomere Length Diversity in Cattle Breeds. Diversity 2010; 2:1118-29

24. Bjørnstad G, Gunby E, Røed KH. Genetic structure of Norwegian horse breeds. Journal of Anim Breed Genet 2000;117:307-317.

25. Aberle KS, Hamann H, Drögemüller C, Distl O. Genetic diversity in German draught horse breeds compared with a group of primitive, riding and wild horses by means of microsatellite DNA markers. Anim Genet 2004; 35:270-277.

26. Vega-Pla JL, Calderon J, Rodriguez-Gallardo PP, Martinez AM, Rico C. Saving feral horse populations: does it really matter? A case study of wild horses from Doñana National Park in southern Spain. Anim Genet 2006; 37: 571-8.

27. Njajou OT, Cawthon RM, Damcott CM, Shih-Hsuan W, Sandy O, Garant MJ, Blackburn EH, Mitchell BD, Shuldiner AR, Hsueh W. Telomere length is paternally inherited and is associated with parental lifespan. PNAS 2007; 104: 12135-12139. 